\title{
Synthesis of Gold Nanoparticles to Capture Lifelike Proteins: Application on the Multichannel Sensor Array Design
}

\author{
Yumin Leng $\mathbb{D},{ }^{1}$ Chunhua Ma, ${ }^{2}$ Wentai Zhang, ${ }^{2}$ Kecheng Liu, ${ }^{3}$ and Zhiwen Lu${ }^{1}$ \\ ${ }^{1}$ College of Physics and Electronic Engineering, Nanyang Normal University, Nanyang 473061, China \\ ${ }^{2}$ College of Mechanical and Electronic Engineering, Nanyang Normal University, Nanyang 473061, China \\ ${ }^{3}$ College of Chemistry and Pharmaceutical Engineering, Nanyang Normal University, Nanyang 473061, China
}

Correspondence should be addressed to Yumin Leng; yumin_leng@126.com

Received 1 August 2017; Revised 26 November 2017; Accepted 5 December 2017; Published 9 January 2018

Academic Editor: Naiqian Zhan

Copyright (C) 2018 Yumin Leng et al. This is an open access article distributed under the Creative Commons Attribution License, which permits unrestricted use, distribution, and reproduction in any medium, provided the original work is properly cited.

\begin{abstract}
The chemical elements of proteins are similar to that of DNA (e.g., C, H, O, and N), and DNA shows different knotted architectures. So we imagine that proteins may show a wealth of highly complex structures, especially when proteins interact with each other. The imagination was proved by synthesizing gold nanoparticles (GNPs) to capture the lifelike protein structures. The optical responses (i.e., color) of as-prepared GNPs are found to be characteristic to a given protein (or heavy metal ion). Based on the "three colors" principle of Thomas Young, we extracted the red, green, and blue (RGB) alterations of as-synthesized GNPs to fabricate multichannel sensor arrays for proteins (or heavy metal ions) discrimination. The designed multichannel sensor arrays demonstrate possibilities in semiquantitative analysis of multiple analytes (e.g., proteins and heavy metal ions). This work is believed to open new opportunities for GNPs-based label-free sensing.
\end{abstract}

\section{Introduction}

Proteins usually fold into one or more specific spatial conformations driven by the noncovalent interactions such as hydrogen bonding, ionic interactions, Van der Waals, and hydrophobic packing [1-3]. Due to the heterogeneous surfaces and numerous spatial configurations, proteins can interact with each other in unpredictable ways [4] and provide opportunities to prepare nanoparticles in the templates of proteins. Interestingly, Taylor comments that protein knots predominantly occur in the catalytic domain or the active site itself [5]. The 2016 Nobel Prize winners, professors Sauvage and Stoddart, predict that advances in the protein area would no doubt continue apace [6]. The chemical elements of proteins are similar to that of DNA (e.g., C, H, O, and N), and DNA shows different knotted architectures [6]. So we imagine that proteins may show a wealth of highly complex structures, especially when proteins interact with each other. To prove such imagination, we designed the experiments to synthesize gold nanoparticles (GNPs) to capture the lifelike protein structures via an in situ reduction method.
Based on this unique optical character, GNPs have been widely employed in colorimetric sensing including metal ions, organic molecules, and proteins [7-10]. The previously reported GNPs-based sensing strategies are mostly designed on the basis of aggregation [11, 12], antiaggregation [13], and etching $[14,15]$ mechanisms, thus specific designs and complicated surface modification of GNPs being generally inevitable. For example, Naik's group developed a peptide functionalized GNPs-based colorimetric sensing method for the detection of heavy metal ions [16]. Valentini et al. described a highly sensitive point mutations probe based on DNA-conjugated GNPs [17]. Beyond these, Xia et al. exploited a facile sensing strategy using single-stranded probe DNA, unmodified GNPs, and conjugated polyelectrolyte to detect multiple targets including biomacromolecules (i.e., nucleic acid and protein), small molecules, and metal ions [18]. Nonetheless, the type of analyte is closely dependent upon the kind of selected probe DNA (i.e., aptamer), and thus their strategy could not veritably achieve simultaneous identification and high throughput detection. Consequently, 
it is still highly desirable and challenging to develop labelfree GNPs-based sensing technology and at the same time to attain simultaneous identification of multiple analytes.

Herein, we are interested to develop label-free GNPsbased sensing arrays for analytes (e.g., proteins and metal ions) discrimination. We employed the in situ reduction method to synthesize GNPs and the "three colors" principle of Thomas Young; that is, "all colors are mixed by red $(\mathrm{R})$, green $(\mathrm{G})$, and blue (B) in different proportions." We further extracted the RGB data (i.e., fingerprints) from the as-synthesized GNP which is corresponding to a certain analyte and fed them into the Multi-Variate Statistical Package (MVSP) computing software [19]. Then the analytes were quantitatively distinguished by using the statistical methods (e.g., principal component analysis (PCA)) [20]. This current work is believed to open a new strategy for GNPs-based sensing without requiring complicated surface functionalization process.

\section{Experimental Section}

2.1. Materials and Instruments. All chemicals were used as received without further purification. Chloroauric acid tetrahydrate $\left(\mathrm{HAuCl}_{4} \cdot 4 \mathrm{H}_{2} \mathrm{O}\right)$, lysozyme (Lys), trypsin (Try), bovine hemoglobin ( $\mathrm{BHb}$ ), pepsin (Pep), and $\mathrm{NaOH}$ were obtained from Sinopharm Chemical Reagent Co., Ltd. (Beijing, China). Collagen (Col), glucoamylase (Glu), and catalase (Cat) were bought from Aladdin Reagent Co. Ltd. (Shanghai, China). Bovine serum albumin (BSA) was purchased from Gibco (Grand Island, USA). 96-well plates (Corning 3632) were obtained from Genetimes Technology. Colorimetric imaging photos were collected by a flatbed scanner (Epson Perfection V300) in 96-well plates. The asprepared GNPs were characterized by transmission electron microscopy (TEM, Tencai F20).

2.2. Preparation of $A u(I)$ Anions. The Col-Au(I) solution was synthesized according to previous reports [21-23]. In brief, $\mathrm{HAuCl}_{4}(1 \mathrm{mM})$ and $\mathrm{Col}(3 \mu \mathrm{g} / \mathrm{mL})$ were incubated at room temperature for 10 min and then mixed with $\mathrm{NaOH}$ solution $(30 \mathrm{mM})$ under vigorous stirring. Thereafter, the colorless solution of $\mathrm{Col}-\mathrm{Au}(\mathrm{I})$ was obtained and stored at $4^{\circ} \mathrm{C}$ for further usage.

2.3. Synthesis of GNPs to Capture the Lifelike Proteins. In the first experiment, $500 \mu \mathrm{L}$ of the as-prepared Col-Au(I) solution was mixed with $15 \mu \mathrm{L}$ proteins $(4 \mathrm{mg} / \mathrm{mL})$ and $200 \mu \mathrm{L} \mathrm{H}_{2} \mathrm{O}_{2}$ $(5 \mathrm{mM})$, then rapidly diluted with water to $6 \mathrm{~mL}$, and mixed vigorously. In the second experiment, $1 \mathrm{~mL}$ of the as-prepared Col-Au(I) solution was mixed with $1 \mathrm{~mL}$ Glu $(4 \mathrm{mg} / \mathrm{mL})$ and subjected to microwave treatment at $300 \mathrm{~W}$ for $90 \mathrm{~s}$. In the third experiment, the $\mathrm{NaOH}(1 \mathrm{ml}, 1 \mathrm{M})$, Glu $(50 \mu \mathrm{L}$, $2 \mathrm{kU} / \mathrm{mL}), \mathrm{HAuCl}_{4}(1 \mathrm{~mL}, 1 \mathrm{mM})$, and heavy metal ions $(20 \mu \mathrm{L}, 80 \mu \mathrm{M})$ were mixed vigorously and then subjected to microwave treatment at $300 \mathrm{~W}$ for $90 \mathrm{~s}$.

2.4. Multichannel Sensor Array for Protein Discrimination. The colorless Col-Au(I) solution in the absence and presence of proteins and $\mathrm{H}_{2} \mathrm{O}_{2}$ were loaded into a 96-well plate, respectively. The flatbed scanner and photoshop software were used to acquire the $\triangle \mathrm{RGB}$ alterations of the control (in the absence of proteins and $\mathrm{H}_{2} \mathrm{O}_{2}$, denoted as "before") and the work solutions (in the presence of proteins and $\mathrm{H}_{2} \mathrm{O}_{2}$, denoted as "after"). The $\triangle$ RGB data (see Table S1, Electronic Supplementary Material (ESM)) were fed into MVSP (Kovach Computing software) [19]. Then the different proteins were quantitatively distinguished by using the statistical methods (e.g., PCA and and hierarchical clustering analysis (HCA)) $[19,20,24]$.

2.5. Multichannel Sensor Array for Heavy Metal Ions Discrimination. The Glu and $\mathrm{HAuCl}_{4}$ mixture in the absence and presence of heavy metal ions were subjected to microwave heating at $300 \mathrm{~W}$ for $90 \mathrm{~s}$ and then loaded into a 96-well plate, respectively. The flatbed scanner and photoshop software were used to acquire the $\triangle \mathrm{RGB}$ alterations of the control (in the absence of heavy metal ions, denoted as "before") and the work solutions (in the presence of heavy metal ions, denoted as "after"). The $\triangle$ RGB data (see Table S2, ESM) were fed into MVSP, and the different heavy metal ions were discriminated by PCA and HCA $[19,20,24]$.

\section{Results and Discussion}

3.1. Synthesis of GNPs to Capture the Lifelike Protein Structures. The chemical elements of proteins are similar to that of DNA (e.g., $\mathrm{C}, \mathrm{H}, \mathrm{O}$, and $\mathrm{N}$ ), and DNA shows different knotted architectures [6]. So we imagine that proteins may show a wealth of highly complex structures, especially when proteins interact with each other. To prove such imagination, we designed the experiments to synthesize GNPs in the protein systems via an in situ reduction method. The selected proteins (e.g., Col, Glu, Pep, BHb, Lys, Try, and BSA) are closely related to the life of humans and animals. The multiple interactions among different proteins can induce them showing multiple spatial structures in different environment. It is expected that the multiple spatial structures can be solidified by using strong alkaline or rapid heating. At the same time, the solidified proteins would be covered with GNPs.

The First Experiment. As shown in Figure 1, the GNPs synthesis involves the chemical reduction of $\mathrm{Au}(\mathrm{III}) \rightarrow \mathrm{Au}(\mathrm{I})$ using $\mathrm{Col}$ in the strong alkaline solution and $\mathrm{Au}(\mathrm{I}) \rightarrow \mathrm{Au}(0)$ in the presence of $\mathrm{H}_{2} \mathrm{O}_{2}[21,25,26]$, rapidly producing GNPs assembled/overgilt on the protein structures. In detail, $\mathrm{Au}$ (III) (i.e., $\left[\mathrm{AuCl}_{4}\right]^{-}$, light-yellow) was firstly reduced by $\mathrm{Col}$ to obtain $\mathrm{Au}(\mathrm{I})$ solution (i.e., $\left[\mathrm{AuCl}_{2}\right]^{-}$, colorless) [2123]. Then seven proteins were selected as testing model to verify the imagination of the lifelike activities of proteins. As shown in Figure $\mathrm{S} 1$ (ESM), upon the addition of $\mathrm{H}_{2} \mathrm{O}_{2}$ to the mixtures of $\mathrm{Col}-\mathrm{Au}(\mathrm{I})$ and tested proteins, the colorless solution of $\mathrm{Col}-\mathrm{Au}(\mathrm{I})$ obviously changed into various colors due to the rapid formation of GNPs on the surfaces of different proteins. It is worth mentioning that the colors of GNPs corresponding to BHb, BSA, and Lys show the different shades of blue (Figure S1, ESM), which means that 


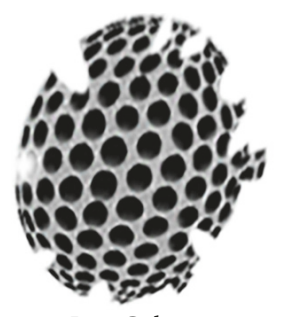

Pep-Col

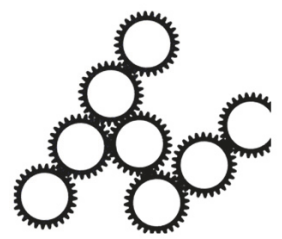

Cat-Col

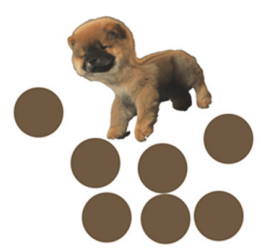

BSA-Col

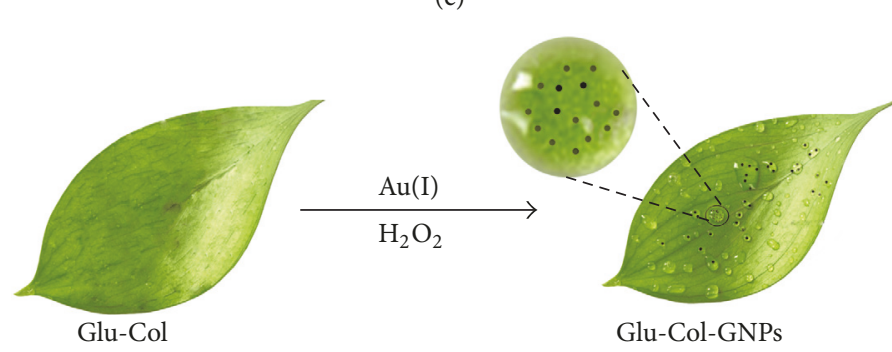

(d)

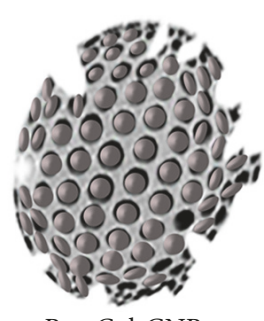

Pep-Col-GNPs

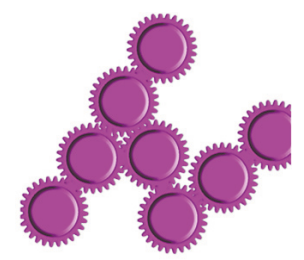

Cat-Col-GNPs

(b)

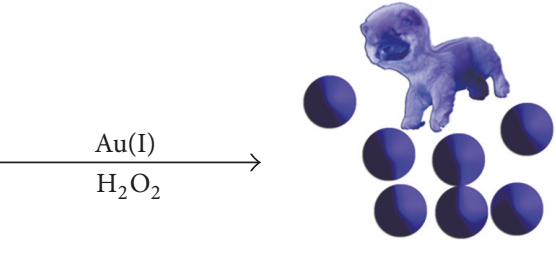

BSA-Col-GNPs (c)

1. Schematic illustration of GNPs synthesis to capture the lifelike protein structures. The multistructures (e.g., irregular porous balls, gears, animal actions, and leaves) may exist in the mysterious protein systems. To verify the imagination, we synthesized GNPs in the proteins solution via the in situ reduction of $\mathrm{Au}(\mathrm{I})$ using $\mathrm{H}_{2} \mathrm{O}_{2}$. The imaginary structures are formed by simple mixing Pep, Cat, BSA, Glu, and Col, respectively.

the as-synthesized GNPs may be similar to each other or aggregative. To prove the conjectures, we characterized the as-prepared GNPs by TEM. As shown in Figure 2, the GNPs show the different shapes, such as particles dispersed in the irregular porous balls, gears, animal actions, and spheres assembled on leaves. Interestingly, all the shapes of GNPs corresponding to BSA, BHb, and Lys present dog actions, as shown in Figure 2(c) and Figure S2 (ESM). The representative UV-vis absorption spectra were further characterized to reveal the reason for GNPs exhibiting different colors. As shown in Figure S3 (ESM), the UV-vis absorption intensities of as-synthesized GNPs are different and the corresponding peaks appear at $\sim 570 \mathrm{~nm}$ (Try), $605 \mathrm{~nm}$ (BSA and BHb), and $640 \mathrm{~nm}$ (Lys), which means that the produced GNPs are of different concentrations and sizes. The blue coloration of GNPs corresponding to BSA, BHb, and Lys should be more aggregated than the red coloration of GNPs corresponding to Try.

The Second Experiment. By adding $\mathrm{H}_{2} \mathrm{O}_{2}$ to the mixtures of Glu and Col-Au(I), we synthesized gold nanospheres on leaves (see Figure 2(d)). In particular, interestingly, the mixture of Glu and Col-Au(I) was then subjected to microwave treatment. The $\mathrm{Au}(\mathrm{I})$ ions could be reduced by proteins to be GNPs [21], which might be dispersed on the rolled leaves (as shown in Figure 3(a)). The imagination is proved by the TEM characterization (see Figure 3(b)). The captured structure of Glu-Col (see Figure 3(b)) is similar to the peptide 


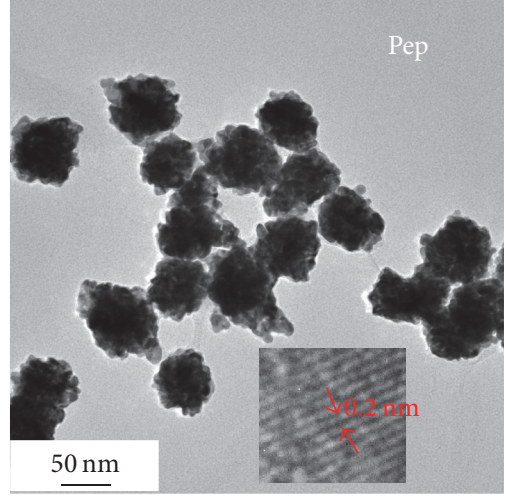

(a)

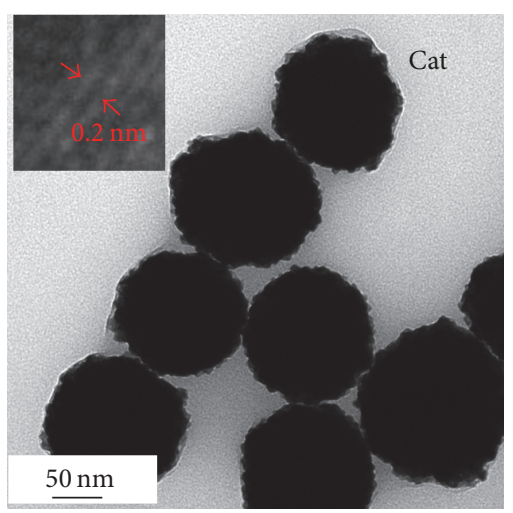

(b)

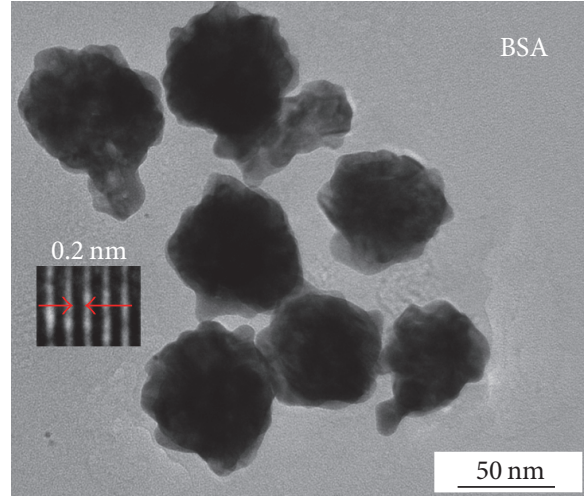

(c)

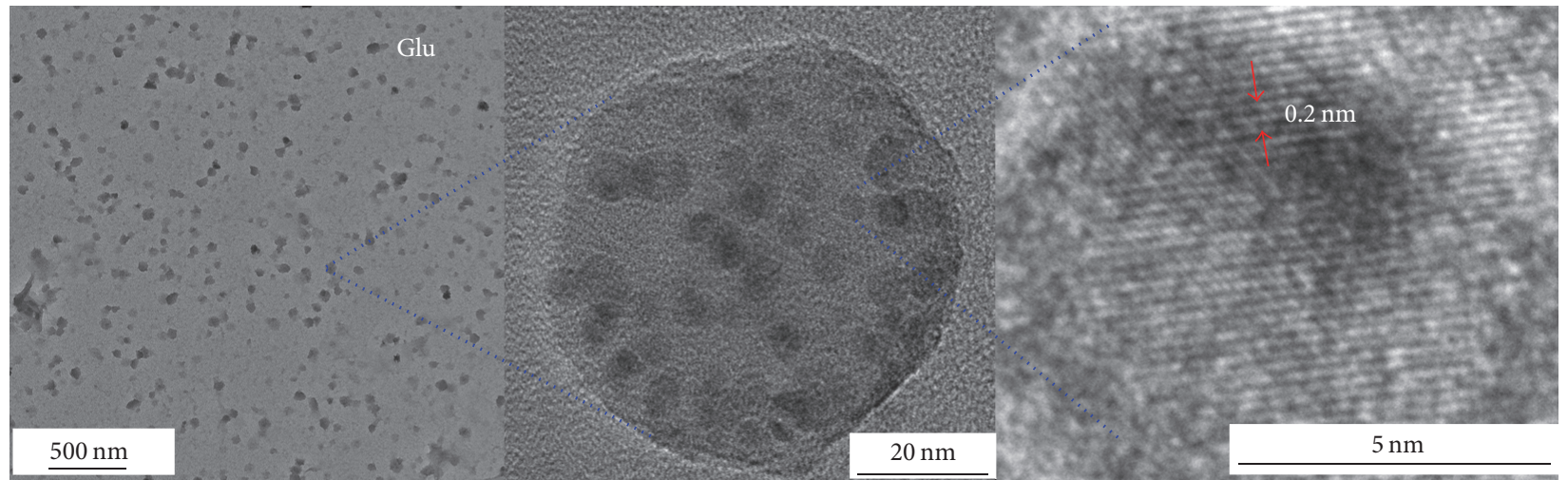

(d)

FIGURE 2: TEM and high resolution TEM (HRTEM) images of GNPs obtained by the in situ reduction of Au(I) to GNPs in the protein systems (the signed proteins interacted with $\mathrm{Col}$ ).

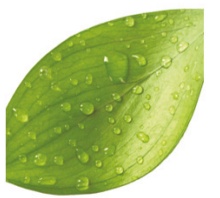

Glu-Col-Au(I)

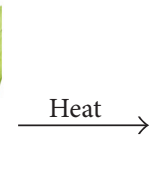

(a)

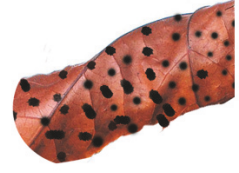

Glu-Col-GNPs

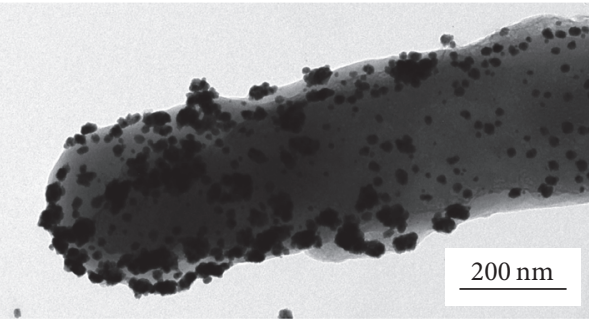

(b)

Figure 3: (a) Schematic illustration of GNPs synthesis via microwave heating the mixture of Glu and Col-Au(I) solution (Glu-Col-Au(I)). (b) The corresponding TEM image of GNPs.

configuration reported by professor Feringa (the 2016 Nobel Prize winner) [4].

The Third Experiment. Inspired by professor Sauvage's theory of "In the presence of metal ions, molecules can form the double-stranded double helix" [27], we designed another experiment to capture the life actions in protein systems (see Figure 4 ). In the strong alkaline solution, $\left[\mathrm{AuCl}_{4}\right]^{-}$ ions were mixed with certain heavy metal ions (e.g., $\mathrm{Mn}^{2+}$, $\mathrm{Co}^{2+}, \mathrm{Cu}^{2+}, \mathrm{Cr}^{3+}, \mathrm{Ni}^{2+}, \mathrm{Hg}^{2+}, \mathrm{Zn}^{2+}, \mathrm{Pb}^{2+}$, and $\left.\mathrm{Cr}(\mathrm{VI})\right)$ at a molar ratio of $625: 1$ and then mixed with the protein of
Glu rapidly. According to our previous reports [21, 23], the metal ions should be reduced to monovalent metal ions (e.g., $\mathrm{Au}(\mathrm{I}), \mathrm{Mn}(\mathrm{I}), \mathrm{Co}(\mathrm{I})$, and $\mathrm{Cu}(\mathrm{I}))$. Livoreil et al. forecasted that the metal-mediated interlocking rings could in principle be extended to polymers [28], and the macromolecular systems incorporating interlocking rings would lead to interesting systems [29]. Here, the monovalent metal ions may mediate the amino acid residues present in Glu to be interlocking rings (i.e., double-stranded double helices) [23, 27]. As shown in Figure S4 (ESM), the strong interactions between monovalent metal ions and the functional groups of Glu (e.g., 


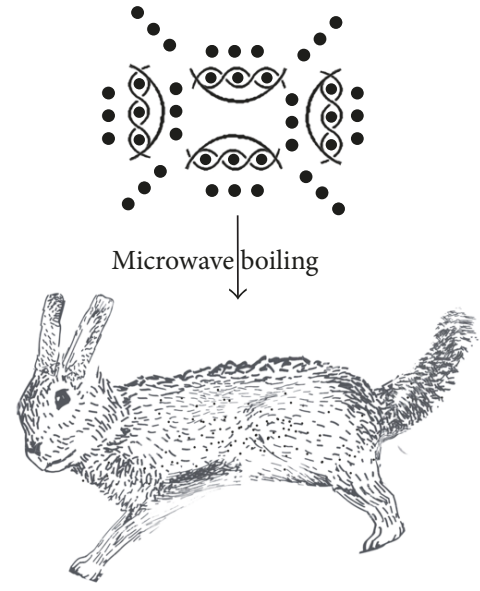

(a)

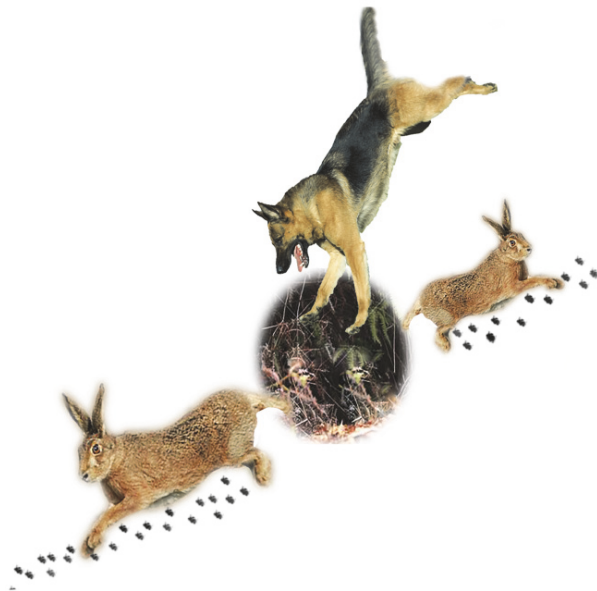

(b)

FIGURE 4: (a) Schematic illustration of animal-hair-shaped GNPs synthesis via microwave boiling the mixtures of metal ions, Au(III), and Glu solution. The double-stranded double helixes in the protein system are directed by metal ions. (b) The life actions are imagined to exist in the biological fields.

$-\mathrm{NH}$ and $-\mathrm{CO}$ ) help the metal ions in rings to be the cores, which could be deposited by the free metal ions. Through the rapid nucleation method, the metal ions (mainly $\mathrm{Au}(\mathrm{I})$ ions) were reduced by the reductive proteins to be GNPs [21]. The animal-hair-shaped GNPs would be synthesized via microwave boiling the helices. The animal actions (see Figure 4(b)) are expected to be captured to synthesize GNPs in the protein systems. The helices containing different distribution and number of various metal ions would result in the synthesized GNPs exhibiting multicolors. The mixtures of metal ions, $\mathrm{Au}(\mathrm{III})$ and Glu solution, were subjected to microwave treatment at $300 \mathrm{~W}$ for $90 \mathrm{~s}$, and the GNPs of distinguishable colors were synthesized. By adding $\mathrm{Hg}^{2+}$ and $\mathrm{Mn}^{2+}$ to the mixture of $\left[\mathrm{AuCl}_{4}\right]^{-}$and Glu, we captured the animal actions. As shown in Figure 5(a), a dog is pouncing to the rabbits' hole; the rabbit mother and cub are escaping and leaving their foot prints (see Figures 5(c) and 5(d)). As shown in Figure 5(b), the as-synthesized GNPs are similar to the hairs of animals (e.g., rabbit and dog). The insets of Figure 2, Figure S2 (SI), and Figures 5(b) and 5(d) show that the interplanar distances of GNPs are approximately $0.2 \mathrm{~nm}$, which can be indexed to $\{200\}$ plane of gold [30]. All the captured protein structures show that the matter, movements, and life actions exist in the biological fields.

\subsection{Application on Multichannel Sensor Array Design}

Protein Discrimination. Encouraged by the distinct colors of GNPs (Figure S1, ESM), we expect that the Col-Au(I) solution might be a promising probe for constructing "single-indicator-based multidimensional sensing" platform that we established recently [31], for the discrimination of proteins. According to the "three colors" principle of Thomas Young, we extracted the RGB alterations of the singleindicator-based sensor images, "before" and "after" exposure to proteins and $\mathrm{H}_{2} \mathrm{O}_{2}$ (Figure $\mathrm{S} 1$ and Table S1, ESM). The
RGB alterations provide diverse fingerprints, as shown in Figure 6(a). The $\triangle$ RGB data are further fed into the MVSP computing software and classified by statistical methods, that is, PCA and HCA $[19,20,24]$. As shown in Figure 6(b), the results of PCA prove that the canonical response patterns of proteins are clearly clustered into different groups, significantly separating from the control. The results of HCA show that all of the 40 cases ( 8 samples $\times 5$ replicates) are correctly assigned to their respective groups (Figure S5, ESM). The constructed platforms provide a new way to detect the proteins contained in the complex matrix (e.g., milk), showing great promise for milk safety in the future.

Heavy Metal Ions Discrimination. As shown in Figure 7, from the color images of the $\left[\mathrm{AuCl}_{4}\right]^{-}$and Glu mixture (as indicator) in the absence (named "before") and presence (named "after") of heavy metal ions after microwave reaction, the obvious color differences are observed to the target heavy metal ions. The visual sensor array seems to be successful at differentiating the heavy metal ions $(0.77 \mu \mathrm{M})$ as shown in the difference maps, which are obtained through taking the RGB variations from the "before" and "after" images. The unique pattern of difference maps is corresponding to a certain heavy metal ion (see Figure 7). The observations demonstrate that the single-indicator-based sensor can potentially determine the target heavy metal ions $(0.77 \mu \mathrm{M})$ via constructing the multichannel visual sensing array.

The RGB alterations (Table S2, ESM) provide diverse fingerprints (see Figure 8(a)), further fed into MVSP computing software and classified by PCA $[19,20]$. The canonical colorimetric response patterns ( 3 channels $\times 10$ samples $\times 4$ replicates) are clustered into several groups (see Figure 8(b)). The resulting 2D canonical score plot (Figure 8(b)) shows clear clustering of the data using the first two principal components (representing $97 \%$ of the total variance), with excellent discriminatory capacity. The PCA plots for various 


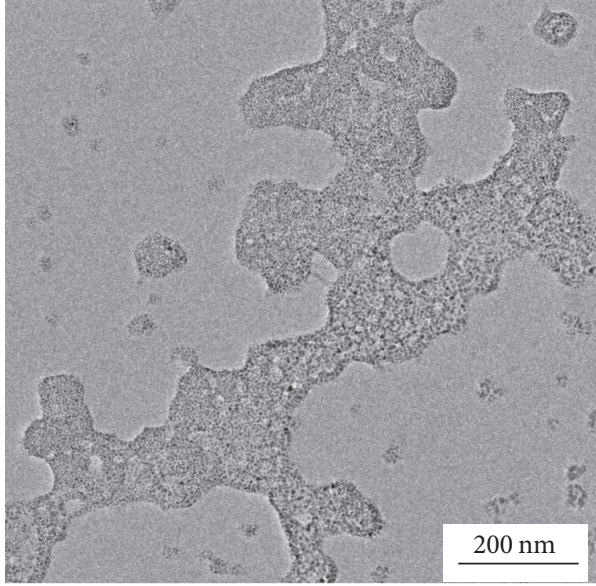

(a)

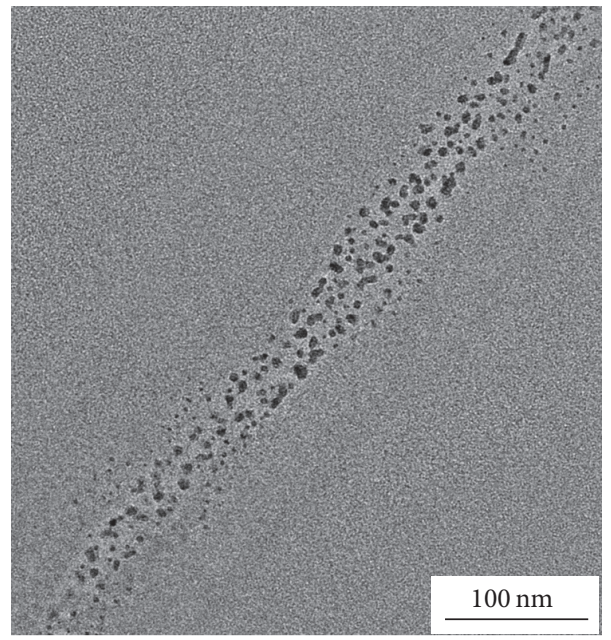

(c)

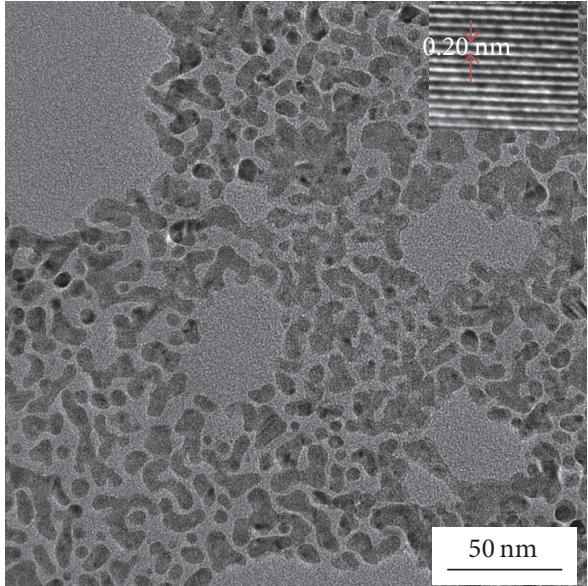

(b)

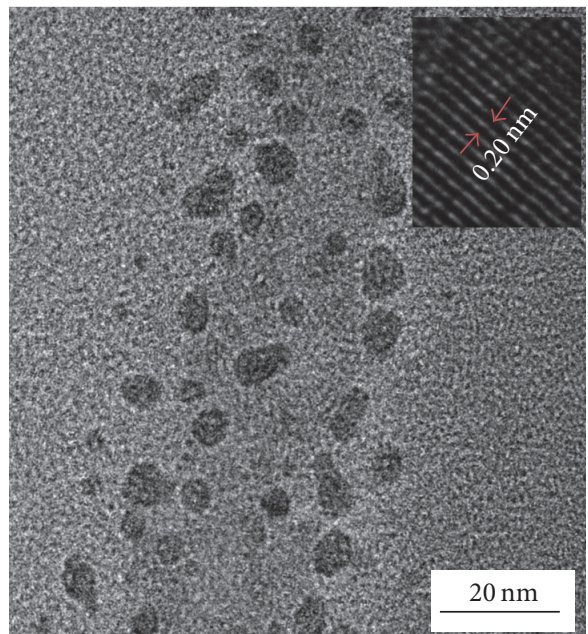

(d)

Figure 5: (a, b) The captured GNPs' TEM images of animal actions and (c, d) foot prints at different magnification. Insets: the interplanar distances of GNPs determined by using ImageJ software.

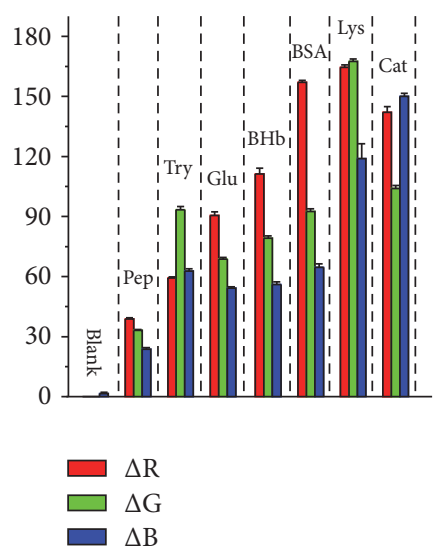

(a)

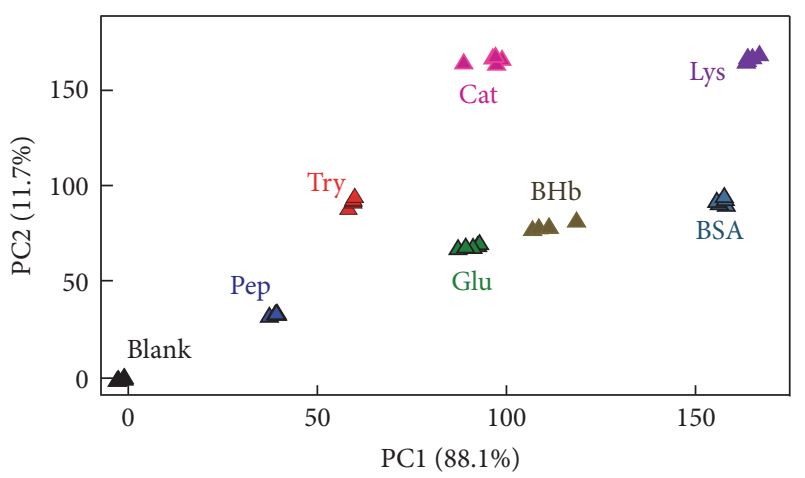

(b)

FIGURE 6: (a) $\triangle$ RGB alterations of the Col- $\mathrm{Au}(\mathrm{I})$ solution in the absence and presence of seven proteins $(10 \mu \mathrm{g} / \mathrm{mL})$ and $\mathrm{H}_{2} \mathrm{O}_{2}$. Each value is an average of five parallel measurements, and the error bars are shown. (b) The seven proteins were quantitatively distinguished by the PCA method. 


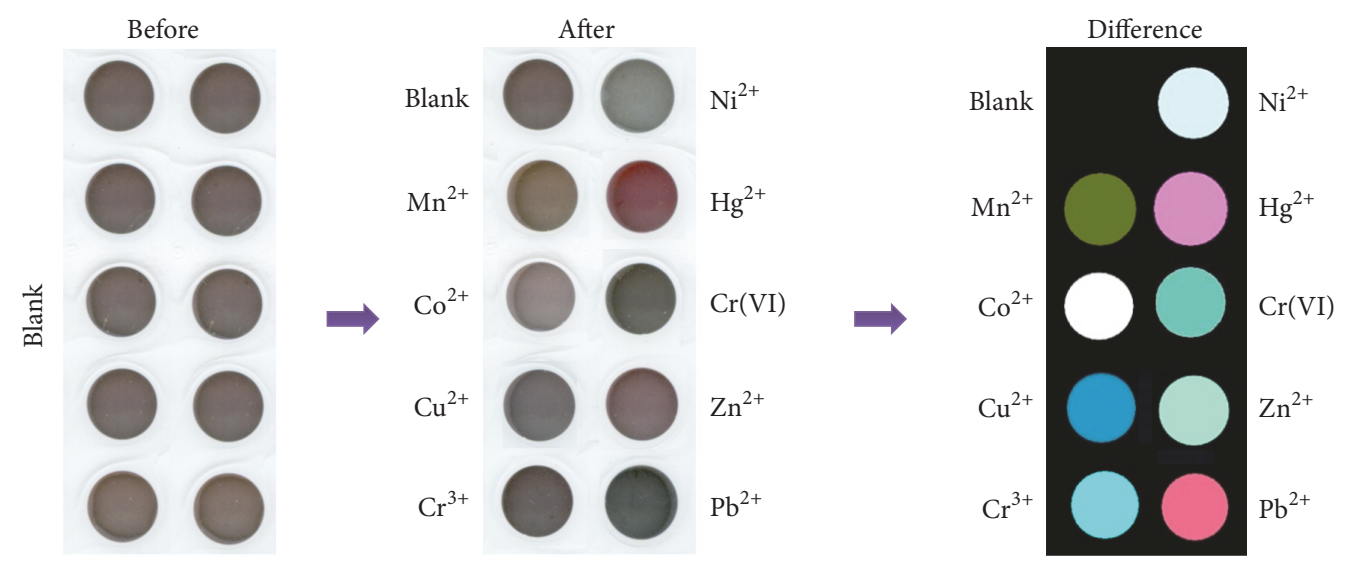

FIGURE 7: Construction of visual sensor array for discrimination of heavy metal ions $(0.77 \mu \mathrm{M})$. Digital photographs of the $\left[\mathrm{AuCl}_{4}\right]^{-}$and $\mathrm{Glu}$ mixture in the absence (named "before") and presence (named "after") of heavy metal ions after microwave reaction. For visualization, the color ranges of these difference maps are expanded from 4 to 8 bits per color (RGB range 4-19 expanded to 0-255).

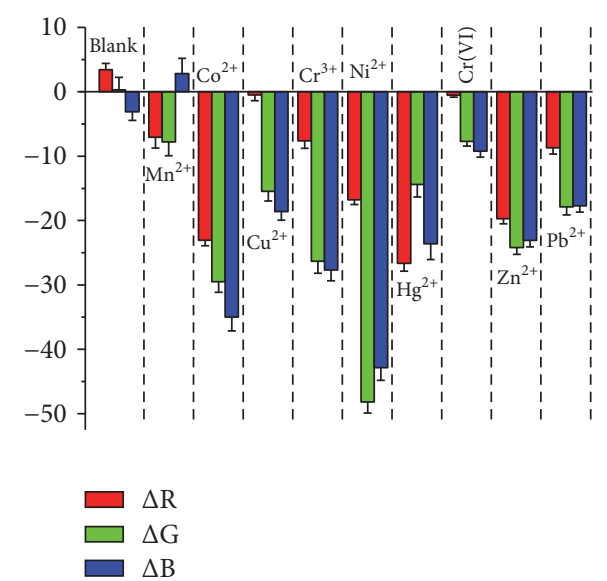

(a)

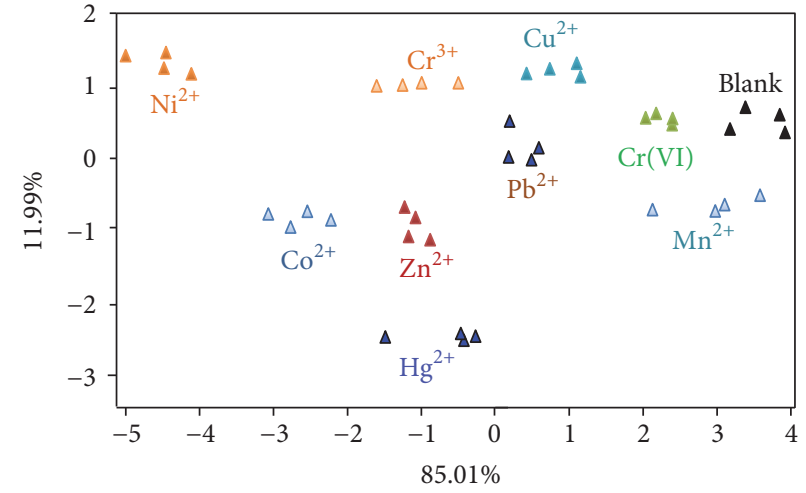

(b)

FIGURE 8: (a) $\triangle \mathrm{RGB}$ alterations of the $\left[\mathrm{AuCl}_{4}\right]^{-}$and Glu mixture in the absence and presence of heavy metal ions $(0.77 \mu \mathrm{M})$ after microwave reaction. Each value is an average of four parallel measurements, and the error bars are shown. (b) The nine heavy metal ions were quantitatively distinguished by the PCA method.

heavy metal ions are not random but rather follow certain patterns. As shown in Figure 8(b), all the target heavy metal ions $(0.77 \mu \mathrm{M})$ are separated from each other, demonstrating that they are effectively discriminated by PCA based on both the $\triangle \mathrm{RGB}$ values (Table S2, ESM). The single indicator in the presence of the nine heavy metal ions at $0.77 \mu \mathrm{M}$ are also analysed using HCA $[19,24]$. All of the 40 cases (10 samples $\times 4$ replicates) are correctly assigned to their respective groups (Figure S6, ESM). The constructed platform provides a new way to detect the heavy metal ions contained in the environmental water or air, showing great promise for environmental safety in the future.

\section{Conclusion}

In summary, the chemical elements of proteins are similar to that of DNA (e.g., C, H, O, and N), and DNA shows different knotted architectures. So we imagined that proteins might show a wealth of highly complex structures, especially when proteins interact with each other. The imaginations have been proved by the experiments of synthesizing GNPs to capture the lifelike protein structures.

Moreover, a novel and facile sensing strategy based on in situ reduction has been developed. The main advantage of this strategy is label-free, which avoided frequently used surface modification in most GNPs-based sensors. The as-proposed sensing method is able to identify and detect multiple analytes (e.g., proteins and heavy metal ions). This work is believed to open new opportunities for fabricating GNPs-based labelfree, simultaneous, and high throughput identification of multiple targets in samples (e.g., milk and environmental water).

\section{Conflicts of Interest}

The authors declare that there are no conflicts of interest regarding the publication of this paper. 


\section{Authors' Contributions}

Yumin Leng conceived the idea, performed the experiments, analysed the data, and wrote the manuscript. Wentai Zhang drew Figures 1, 3(a), and 4. Kecheng Liu characterized GNPs. Chunhua Ma and Zhiwen Lu analysed the data and discussed the paper. All authors have read and approved the manuscript.

\section{Acknowledgments}

This work is supported by the Natural Science Foundation of China (21607083), Natural Science Foundation of Henan (162300410206), Technicians Troop Construction Projects of Henan Province (C20150029), and Scientific and Technological Project of Henan Province (162102310484). Y. Leng thanks Professor Hengwei Lin and Dr. Yuhui Wang for discussions, Kai Jiang for TEM characterization (Figure 3(b)), and Dr. Muhammad Zubair Iqbal for checking the manuscript. Y. Leng also thanks the materials and instrumentation supported by Ningbo Institute of Materials Technology \& Engineering.

\section{Supplementary Materials}

Figure S1: Color images of the Col-Au(I) solution obtained before and after exposure to proteins and $\mathrm{H}_{2} \mathrm{O}_{2}$. The randomly selected proteins $(10 \mu \mathrm{g} / \mathrm{mL})$ are pepsin (Pep), catalase (Cat), bovine hemoglobin ( $\mathrm{BHb})$, bovine serum albumin (BSA), lysozyme (Lys), glucoamylase (Glu), and trypsin (Try). Figure S2: TEM and high resolution TEM (HRTEM) images of GNPs obtained by the rapid reduction of $\mathrm{Au}(\mathrm{I})$ to GNPs, which are overgilt on the protein structures (e.g., $\mathrm{BHb}$ and Lys interacted with $\mathrm{Col})$. Figure S3: The UV-vis absorption spectra of GNPs obtained by the in situ reduction of $\mathrm{Au}(\mathrm{I})$ to GNPs in the protein systems (the signed proteins interacted with Col). Figure S4: Schematic illustration of the interlocking rings directed by monovalent metal ions (M(I)) in Glu. Figure S5: HCA analysis for the discrimination of proteins $(10 \mu \mathrm{g} / \mathrm{ml})$ based on the $\triangle \mathrm{RGB}$ variations. Figure S6: HCA analysis for the discrimination of heavy metal ions $(0.77 \mu \mathrm{M})$ based on the $\triangle \mathrm{RGB}$ variations. Table S1: Database of the $\triangle$ RGB alterations of the Col-Au(I) solution in the absence and presence of seven proteins $(10 \mu \mathrm{g} / \mathrm{ml})$ and $\mathrm{H}_{2} \mathrm{O}_{2}$. Table S2: Database of the $\triangle \mathrm{RGB}$ alterations of the $\left[\mathrm{AuCl}_{4}\right]^{-}$and Glu mixture in the absence and presence of heavy metal ions $(0.77 \mu \mathrm{M})$ after microwave reaction. (Supplementary Materials)

\section{References}

[1] S. Gonen, F. DiMaio, T. Gonen, and D. Baker, "Design of ordered two-dimensional arrays mediated by noncovalent protein-protein interfaces," Science, vol. 348, no. 6241, pp. 13651368, 2015.

[2] O. Keskin, A. Gursoy, B. Ma, and R. Nussinov, "Principles of protein-protein interactions: What are the preferred ways for proteins to interact?" Chemical Reviews, vol. 108, no. 4, pp. 1225$1244,2008$.
[3] O. Keskin, N. Tuncbag, and A. Gursoy, "Predicting protein-protein interactions from the molecular to the proteome level," Chemical Reviews, vol. 116, no. 8, pp. 4884-4909, 2016.

[4] W. Szymański, J. M. Beierle, H. A. V. Kistemaker, W. A. Velema, and B. L. Feringa, "Reversible photocontrol of biological systems by the incorporation of molecular photoswitches," Chemical Reviews, vol. 113, no. 8, pp. 6114-6178, 2013.

[5] W. R. Taylor, "Protein knots and fold complexity: Some new twists," Computational Biology and Chemistry, vol. 31, no. 3, pp. 151-162, 2007.

[6] R. S. Forgan, J.-P. Sauvage, and J. F. Stoddart, "Chemical topology: complex molecular knots, links, and entanglements," Chemical Reviews, vol. 111, no. 9, pp. 5434-5464, 2011.

[7] X. Wei, Z. Chen, L. Tan, T. Lou, and Y. Zhao, "DNA-catalytically active gold nanoparticle conjugates-based colorimetric multidimensional sensor array for proteins discrimination," Analytical Chemistry, vol. 89, no. 1, pp. 556-559, 2016.

[8] S. Wu, D. Li, J. Wang, Y. Zhao, S. Dong, and X. Wang, "Gold nanoparticles dissolution based colorimetric method for highly sensitive detection of organophosphate pesticides," Sensors and Actuators B: Chemical, vol. 238, pp. 427-433, 2017.

[9] Y. Leng, Y. Li, A. Gong, Z. Shen, L. Chen, and A. Wu, "Colorimetric response of dithizone product and hexadecyl trimethyl ammonium bromide modified gold nanoparticle dispersion to 10 types of heavy metal ions: Understanding the involved molecules from experiment to simulation," Langmuir, vol. 29, no. 25, pp. 7591-7599, 2013.

[10] Y. Leng, F. Zhang, Y. Zhang et al., "A rapid and sensitive colorimetric assay method for $\mathrm{Co}^{2+}$ based on the modified $\mathrm{Au}$ nanoparticles (NPs): Understanding the involved interactions from experiments and simulations," Talanta, vol. 94, pp. 271277, 2012.

[11] M. De, S. Rana, H. Akpinar et al., "Sensing of proteins in human serum using conjugates of nanoparticles and green fluorescent protein," Nature Chemistry, vol. 1, no. 6, pp. 461-465, 2009.

[12] W. Sun, Y. Lu, J. Mao, N. Chang, J. Yang, and Y. Liu, "Multidimensional sensor for pattern recognition of proteins based on DNA-gold nanoparticles conjugates," Analytical Chemistry, vol. 87, no. 6, pp. 3354-3359, 2015.

[13] Y.-L. Li, Y.-M. Leng, Y.-J. Zhang, T.-H. Li, Z.-Y. Shen, and A.-G. Wu, "A new simple and reliable $\mathrm{Hg}^{2+}$ detection system based on anti-aggregation of unmodified gold nanoparticles in the presence of O-phenylenediamine," Sensors and Actuators B: Chemical, vol. 200, pp. 140-146, 2014.

[14] Z. Zhang, Z. Chen, D. Pan, and L. Chen, "Fenton-like reactionmediated etching of gold nanorods for visual detection of $\mathrm{Co}^{2+}$," Langmuir, vol. 31, no. 1, pp. 643-650, 2015.

[15] T. A. Dreier and C. J. Ackerson, "Radicals are required for thiol etching of gold particles," Angewandte Chemie International Edition, vol. 54, no. 32, pp. 9249-9252, 2015.

[16] J. M. Slocik, J. S. Zabinski Jr., D. M. Phillips, and R. R. Naik, "Colorimetric response of peptide-functionalized gold nanoparticles to metal ions," Small, vol. 4, no. 5, pp. 548-551, 2008.

[17] P. Valentini, R. Fiammengo, S. Sabella et al., "Goldnanoparticle-based colorimetric discrimination of cancerrelated point mutations with picomolar sensitivity," ACS Nano, vol. 7, no. 6, pp. 5530-5538, 2013.

[18] F. Xia, X. Zuo, R. Yang et al., "Colorimetric detection of DNA, small molecules, proteins, and ions using unmodified gold nanoparticles and conjugated polyelectrolytes," Proceedings of 
the National Acadamy of Sciences of the United States of America, vol. 107, no. 24, pp. 10837-10841, 2010.

[19] R. A. Johnson and D. W. Wichern, Applied multivariate statistical analysis, Prentice Hall, Upper Saddle River, NJ, USA, 6th edition, 2007.

[20] I. T. Jolliffe, "Principal component analysis," Springer Series in Statistics, Springer, New York, NY, USA, 2nd edition, 2002.

[21] Y. Leng, L. Fu, L. Ye et al., "Protein-directed synthesis of highly monodispersed, spherical gold nanoparticles and their applications in multidimensional sensing," Scientific Reports, vol. 6, Article ID 28900, 2016.

[22] C. J. Johnson, E. Dujardin, S. A. Davis, C. J. Murphy, and S. Mann, "Growth and form of gold nanorods prepared by seedmediated, surfactant-directed synthesis," Journal of Materials Chemistry, vol. 12, no. 6, pp. 1765-1770, 2002.

[23] Y. Leng, K. Jiang, W. Zhang, and Y. Wang, "Synthesis of gold nanoparticles from $\mathrm{Au}(\mathrm{I})$ ions that shuttle to solidify: application on the sensor array design," Langmuir, vol. 33, no. 25, pp. 6398-6403, 2017.

[24] S. J. Haswell, "Practical guide to chemometrics," vol. Ch. 4, New York, NY, USA, 2nd edition, 1992.

[25] T. K. Sarma, D. Chowdhury, A. Paul, and A. Chattopadhyay, "Synthesis of Au nanoparticle-conductive polyaniline composite using $\mathrm{H}_{2} \mathrm{O}_{2}$ as oxidising as well as reducing agent," Chemical Communications, no. 10, pp. 1048-1049, 2002.

[26] X. Liu, H. Xu, H. Xia, and D. Wang, "Rapid seeded growth of monodisperse, quasi-spherical, citrate-stabilized gold nanoparticles via $\mathrm{H}_{2} \mathrm{O}_{2}$ reduction," Langmuir, vol. 28, no. 38, pp. 1372013726, 2012.

[27] J.-F. Nierengarten, C. O. Dietrich-Buchecker, and J.-P. Sauvage, "Synthesis of a doubly interlocked [2]-catenane [4]," Journal of the American Chemical Society, vol. 116, no. 1, pp. 375-376, 1994.

[28] A. Livoreil, J.-P. Sauvage, N. Armaroli, V. Balzani, L. Flamigni, and B. Ventura, "Electrochemically and photochemically driven ring motions in a disymmetrical copper [2]-catenate," Journal of the American Chemical Society, vol. 119, no. 50, pp. 12114-12124, 1997.

[29] J.-L. Weidmann, J.-M. Kern, J.-P. Sauvage, Y. Geerts, D. Muscat, and K. Müllen, "Poly[2]-catenanes containing alternating topological and covalent bonds," Chemical Communications, no. 10, pp. 1243-1244, 1996.

[30] H. Yoshida, Y. Kuwauchi, J. R. Jinschek et al., "Visualizing gas molecules interacting with supported nanoparticulate catalysts at reaction conditions," Science, vol. 335, no. 6066, pp. 317-319, 2012.

[31] Y. Leng, S. Qian, Y. Wang et al., "Single-indicator-based multidimensional sensing: detection and identification of heavy metal ions and understanding the foundations from experiment to simulation," Scientific Reports, vol. 6, Article ID 25354, 2016. 


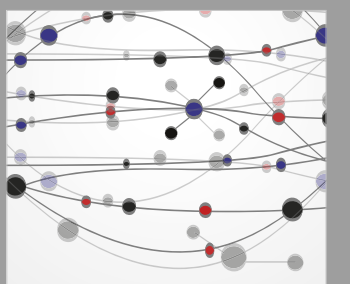

The Scientific World Journal
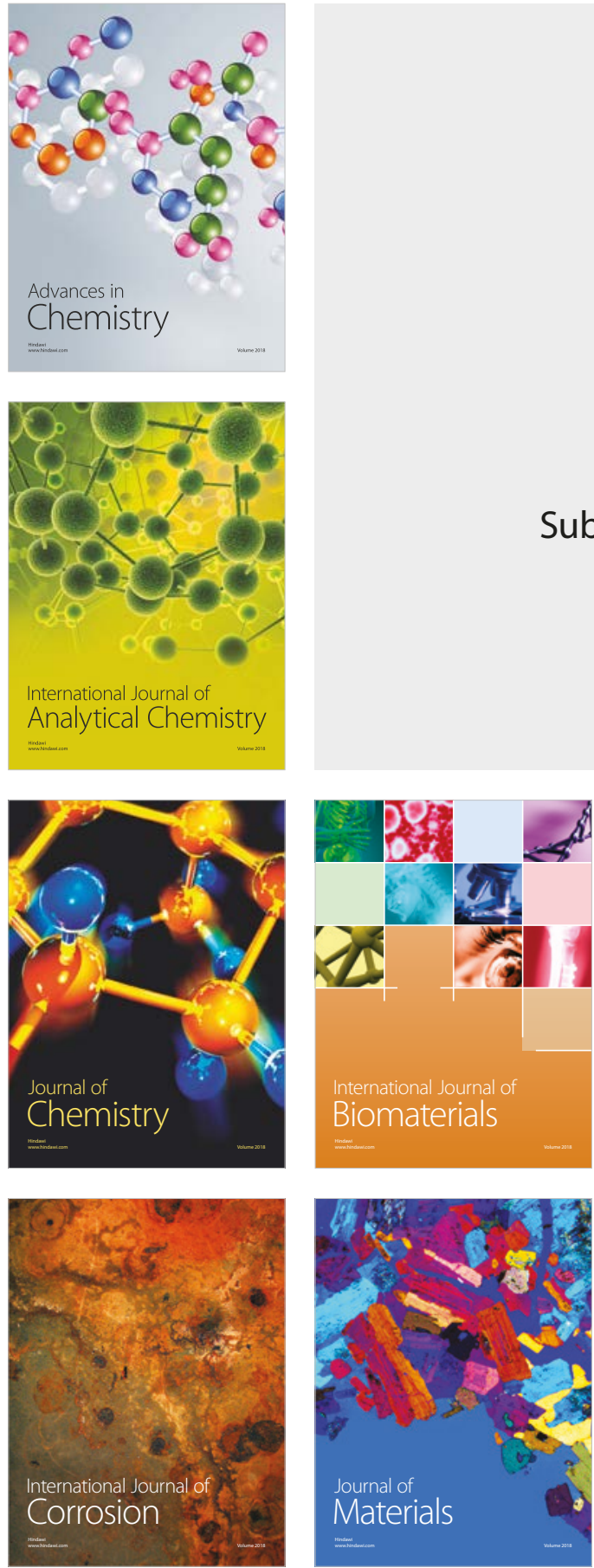

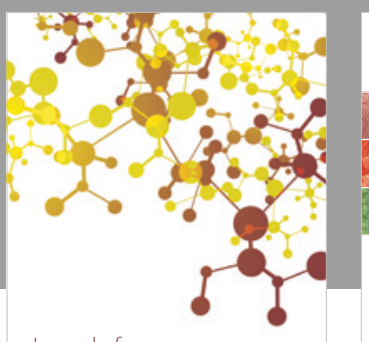

Journal of

Applied Chemistry
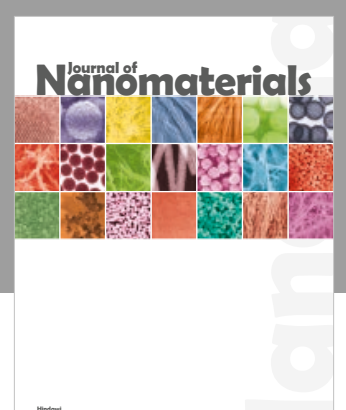

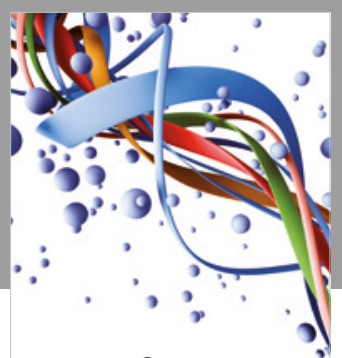

Scientifica

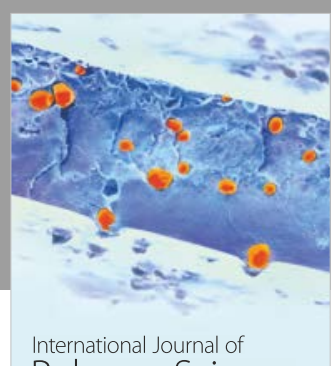

Polymer Science

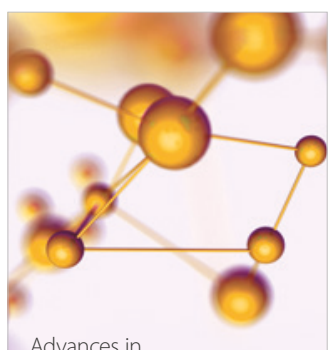

Physical Chemistry
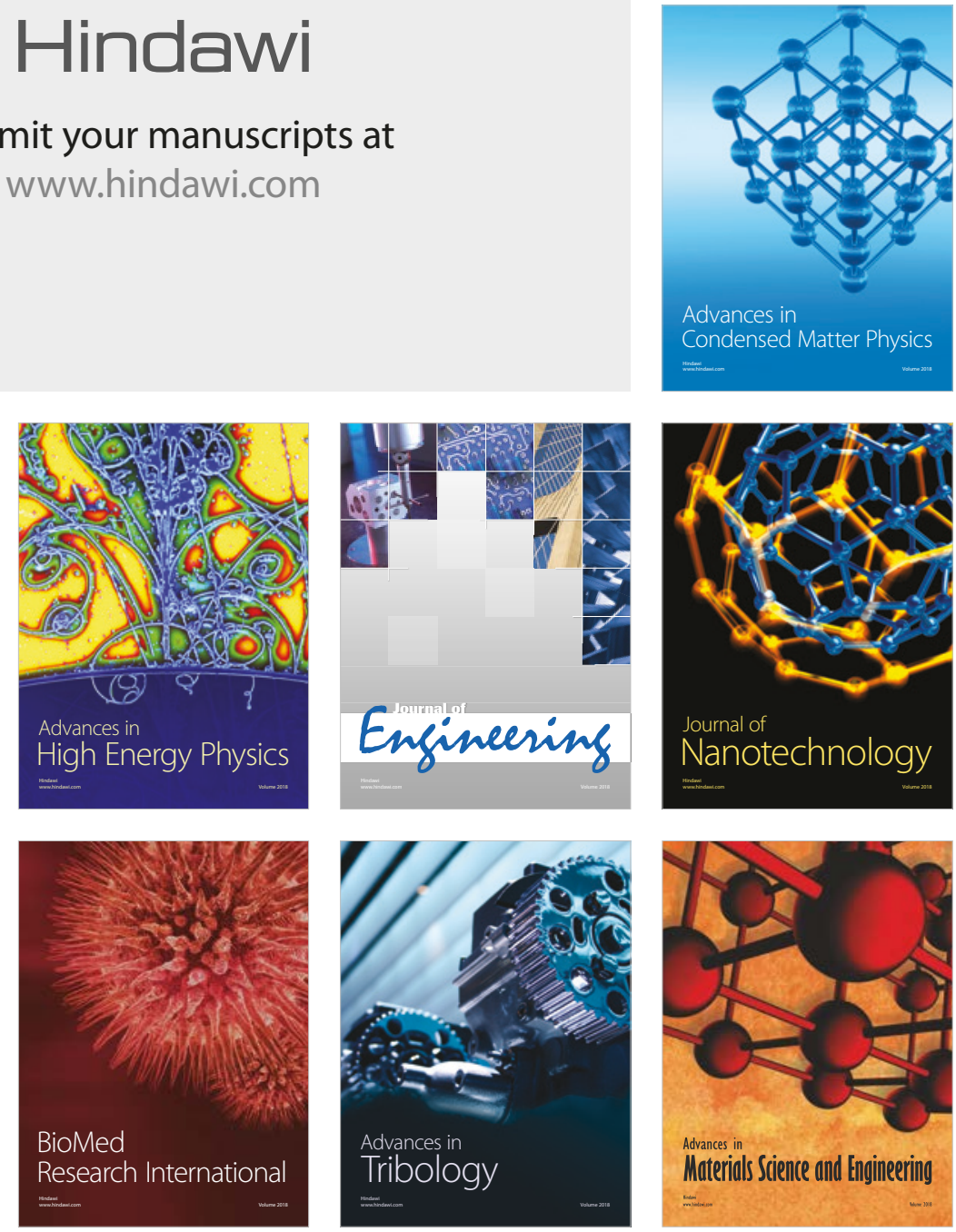